\title{
The Timing Counter of the MEG experiment: calibration and performance
}

\author{
P. W. Cattaneo ${ }^{\text {a * }}$, M. De Gerone ${ }^{\text {b }}$, S. Dussoni ${ }^{\text {b }}$, F. Gatti b, M. Rossella ${ }^{\text {a }}$, Y. Uchiyama ${ }^{\text {c }}$, R. Valle \\ $\mathrm{b}$
}

${ }^{a}$ INFN Pavia, Via Bassi 6, I-27100, Pavia, Italy.

bINFN and Universitá di Genova, Dipartimento di Fisica, Via Dodecaneso 33, I-16146, Genova, Italy

'ICEPP, The University of Tokyo, 7-3-1 Hongo, Bunkyo-ku, Tokyo 113-0033, Japan

The MEG detector is designed to test Lepton Flavor Violation in the $\mu^{+} \rightarrow e^{+} \gamma$ decay down to a Branching Ratio of a few $10^{-13}$. The decay topology consists in the coincident emission of a monochromatic photon in direction opposite to a monochromatic positron. A precise measurement of the relative time $t_{e+\gamma}$ is crucial to suppress the background. The Timing Counter (TC) is designed to precisely measure the time of arrival of the $e^{+}$and to provide information to the trigger system. It consists of two sectors up and down stream the decay target, each consisting of two layers. The outer one made of scintillating bars and the inner one of scintillating fibers. Their design criteria and performances are described.

\section{The MEG experiment}

MEG is an experiment designed to improve significantly the limits on the Branching Ratio (BR) of the $\mu^{+} \rightarrow e^{+} \gamma$ decay [1]-[2]. This decay channel is strongly suppressed in the Standard Model but is allowed to a measurable level in many alternative theories 3 .

The topology of this decay is very simple, consisting of monochromatic $e^{+}$and $\gamma$ with a common space time origin, with energy close to half the $\mu^{+}$mass, moving in opposite directions. These features can be summarized as

$$
\begin{aligned}
& \text { - } E\left(e^{+}\right)=E_{e^{+}}^{s i g} \approx m_{\mu^{+}} / 2 \\
& \text { - } E(\gamma)=E_{\gamma}^{s i g} \approx m_{\mu^{+}} / 2 \\
& \text { - } T_{e^{+} \gamma}=T_{e^{+}}-T_{\gamma}=0 \\
& \text { - } \bar{v}_{e^{+}}=\bar{v}_{\gamma} \\
& \text { - } \Theta_{e^{+} \gamma}=\Theta_{e^{+}}-\Theta_{\gamma}=\pi
\end{aligned}
$$

where $\bar{v}$ is the origin space vertex, $T$ the origin time, $\Theta$ the relative polar angle and $E$ the energy. The main difficulty in this measurement is that,

\footnotetext{
*Corresponding author: Paolo.Cattaneo@pv.infn.it
}

in order to collect the high statistic required to improve the existing $\mathrm{BR}$ in a few years, a very high rate of $\mu^{+}$must decay on the target: $\approx 310^{7} \mathrm{~Hz}$ is the design value. The Michel decay $\mu^{+} \rightarrow e^{+} \nu \bar{\nu}$ produces $e^{+}$with an energy up to $E_{e^{+}}^{s i g}$, therefore high resolution energy measurement is required to reduce the Michel $e^{+}$contamination.

The $\gamma$ background originates from radiative decay (RD) $\mu^{+} \rightarrow e^{+} \gamma \nu \bar{\nu}, e^{+} e^{-}$annihilation in flight and $e^{+}$bremsstrahlung. The kinematic limit is $E_{\gamma^{+}}^{s i g}$ and therefore background suppression needs high resolution measurement of $E(\gamma)$.

The $\gamma$ and $e^{+}$directions and the $e^{+}$vertex need to be measured with high precision to discriminate combinatorial background from random association of $e^{+}$and $\gamma$ from different $\mu^{+}$decays. Precise measurements of $T_{e^{+}}$and $T_{\gamma}$ reduce the combinatorial background from different $\mu^{+}$decays.

The photon detector is a Liquid Xenon Calorimeter (LXe) exploiting scintillation light [8] that provides a measurement of the photon energy, position and timing.

The positron is observed by a spectrometer having at the core a set of 16 low mass Drift Chambers (DCH) embedded in a high magnetic field 
$(\mathcal{O}(1 T))$ with a gradient along the $\mathrm{z}$ axis to bend the positron with a radius weakly dependent on the emission polar angle. The DCH measures the $e^{+}$momentum and position but is unable to deliver trigger information and to provide precise timing [7. The trigger and timing information on the $e^{+}$are delivered by the Timing Counter, that is described in detail in the following.

\section{The Timing Counter: the design}

The TC is required to cover the solid angle opposite to the LXe providing high efficiency for $e^{+}$detection. This requirement is satisfied dividing the TC in two modules, called sectors, placed symmetrically with respect to the decay target. Each sector must provide a precise measurement of the $e^{+}$timing both at the trigger and analysis levels $(\mathcal{O}(100 p s))$. Furthermore it provides a measurement of the crossing point both for the trigger, requiring the $e^{+}$and $\gamma$ moving in opposite direction, and for the analysis, DCH-TC match and determination of the $e^{+}$track path length. These requirements are met with a two layer detector: the outer one measures the transverse coordinate ( $\phi$ with respect to the beam direction) and provides in addition time information for trigger and analysis. The inner one measures the longitudinal coordinate ( $z$ along the beam direction) providing trigger and analysis information.

\subsection{The Longitudinal Timing Counter}

The outer layer consists of 15 scintillator bars located along the z-axis at fixed radius $(\approx 30 \mathrm{~cm})$ in a barrel-like array with $10.5^{\circ}$ gap (see Fig.1). This configuration has high acceptance for $\mu^{+} \rightarrow$ $e^{+} \gamma$ decay with momentum $p_{e^{+}}=52.83 \mathrm{MeV} / \mathrm{c}$ and reduced acceptance for Michel events. The number of bars is matched to the number of $\mathrm{DCH}$ and to the trigger requirement for selecting collinear $e^{+}-\gamma$. The bars have a square section with edge $4.0 \mathrm{~cm}$ and length $80 \mathrm{~cm}$ and are read by fine-mesh PMTs adequate for use in high intensity magnetic field. The criteria leading to the choice of these parameters are explained in [4]. The signals from the PMTs are processed by a Double Threshold Discriminator (DTD) with a low threshold to reduce Time Walk effect and a high threshold to remove background events. When the DTD is fired, it delivers a NIM signal. The PMT and the NIM signals are read by the Domino Ring Sampler a custom designed digitizer operating in MEG at frequency up to $2 \mathrm{GHz}[5$. The full waveforms are stored so that the time can be extracted offline with optimized algorithms.

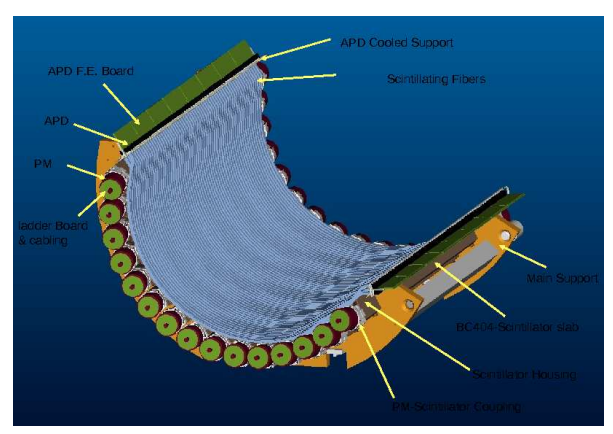

Figure 1. The MEG timing counter

\subsection{The Transverse Timing Counter}

The inner layer is made of $2565 \times 5 \mathrm{~mm}^{2}$ scintillating fibers providing trigger and analysis information on the $z$ coordinate [6] (see Fig, 1). Each fiber is read out by APDs (Avalanche Photo Diode) that allow operations in high intensity transverse magnetic field with a gain $\approx 10^{3}$.

The analog signal from the APD are filtered, 16 of them are summed and made available to the trigger. In parallel, the signals are discriminated and the digital hitmap is made available for the analysis.

\section{The Timing Counter: calibration and performances}

The longitudinal layers started taking data in 2006 and have been active during the following years. To achieve the design resolution, it turned out necessary to calibrate carefully the detector. The calibration tools naturally available are the 
large flux of Michel $e^{+}$from $\mu^{+}$decays and the cosmic rays. There are two other calibration sources requiring a dedicated set up. A $\pi^{-}$beam impinging on a liquid $H$ target produces through charge exchange reaction Dalitz decays $\pi^{0} \rightarrow \gamma e^{+} e^{-}$. Protons of kinetic energy $T \approx 1 \mathrm{MeV}$ from a custom Cockroft-Walton accelerator produces on a Boron target the nuclear reaction ${ }_{5}^{11} B(p, 2 \gamma)_{6}^{12} C$.

Important calibration items are the Time Walk (TW) terms that account for the amplitude dependence of the timing measurement. They are measured using $e^{+}$hitting two or three bars. The same events are used to measure the TC time resolution.

Other crucial calibration is the measurement of timing offset of the different PMTs to allow combining results from different part of the TC. This calibration is obtained with the Dalitz or Boron events, that have two particles emitted at the same time, using the LXe as reference.

The single bar time resolutions with TW correction and offset subtracted are shown in Fig.2. The transverse layer worked only partially due to problems with the digital readout and excess noise, that prevented an efficient use of the detector. With the data available, it was possible to verify that the tracks reconstructed from $\mathrm{DCH}$ match the hits on both transverse and longitudinal layers reducing the combinatorial background and delivering improved information on the $e^{+}$ track length.

\section{Conclusions}

The TC of MEG has performed as high resolution timing detector according to the expectations playing a crucial role in the MEG trigger system. The transverse layer met unexpected problems with part of the digital readout, but the working part delivered satisfactory results. For the 2010 run, both layers are expected to be fully operational.

\section{REFERENCES}

1. T. Mori et al., The MEG experiment: search for the $\mu^{+} \rightarrow e^{+} \gamma$ decay at PSI, May 1999

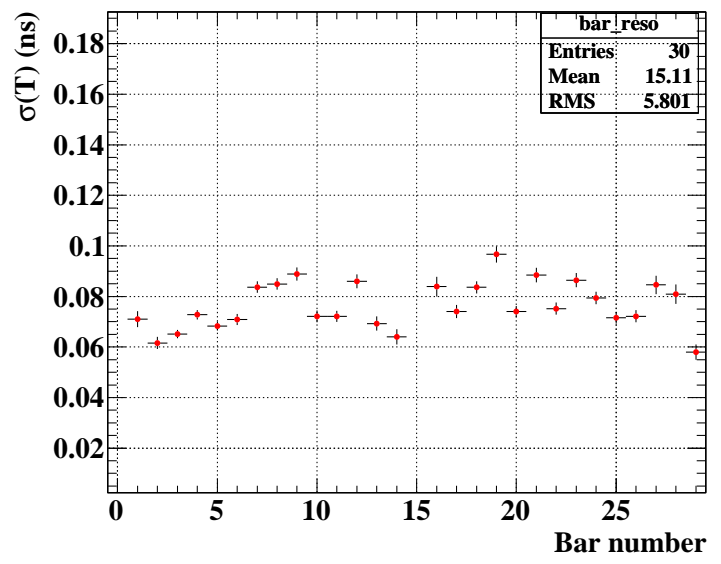

Figure 2. The single bar time resolution using two-bar coincidence.

(http://meg.web.psi.ch/docs/index.html)

2. A. Baldini et al., The MEG experiment: search for the $\mu^{+} \rightarrow$ $e^{+} \gamma$ decay at PSI, May 2002 (http://meg.web.psi.ch/docs/index.html)

3. P. W. Cattaneo, Nucl. Physics B (Proceedings Supplements) 188 (2009) 351-353

4. S. Dussoni et al., Nucl. Instr. and Meth. A 617 (2010) 387-390

5. S. Ritt, IEEE Trans. Nucl. Sci. 4 (2007) 2485

6. M. De Gerone et al., Nucl. Instr. and Meth. A 610 (2009) 218-221

7. P.W. Cattaneo, Nucl. Instr. and Meth. A (2010), doi:10.1016/j.nima.2010.02.245

8. R. Sawada, Nucl. Instr. and Meth. A (2010), doi:10.1016/j.nima.2010.02.214 